\title{
A DISTORTION CORRECTION METHOD FOR ENDOSCOPE IMAGES BASED ON CALIBRATION PATTERNS AND A SIMPLE MATHEMATIC MODEL FOR OPTICAL LENS
}

\author{
Chieh-Hao Hsu, Shaou-Gang Miaou, Feng-Ling Chang \\ Multimedia Computing and Telecommunication Lab., Department of Electronic Engineering, \\ Chung Yuan Christian University, Chung-Li, Taiwan
}

\begin{abstract}
In the past 30 years, the progress in optical engineering, computer science and electronic techniques have made the endoscopy an invaluable tool in both internal clinics and surgical operation. As its applications increase exponentially, it has even become a specialized division in the clinical medicine. In order to obtain a larger field of view inside a small and narrow gastrointestinal tract, an endoscope is usually equipped with a wide-angle lens (Fish eye lens). Thus, an acquired image is often with certain degree of shape distortion. The distortion gets even more serious as the objects extend outward from the center of the lens in radial. This paper discusses the effect of such distortion and the correction of the effect. By using a calibration pattern, the nonlinear distortion is corrected with a simple mathematic model for the endoscope image. Once the endoscopic lens is calibrated, the same mathematic model can be utilized repeatedly for the images captured by that endoscope.

After capturing the calibration pattern using an endoscopic instrument, digital image processing techniques are applied to extract the calibration pattern from the distorted image. We propose a second order mathematic model and consider the parameters of optical lens. The coordinates of each dot in the calibration pattern are the input to the mathematic model for the correction of endoscope images. The experimental results show that the correction method is effective. For example, by comparing to the original calibration pattern image, the average errors in area calculation are $76.46 \%$ and $4.68 \%$ for the distorted and the corrected images, respectively.
\end{abstract}

Biomed Eng Appl Basis Comm, 2005(December); 17: 309-318.

\section{INTRODUCTION}

The application of endoscopy to medicine is quite common. In pulmonary medicine, urology, gynecology, even orthopedic [1], the endoscope is

Received: July 20, 2005; Accepted: Oct. 25, 2005

Correspondence: Shaou-Gang Miaou, Professor

Department of Electronic Engineering, Chung Yuan

Christian University, Chung-Li, Taiwan

E-mail: miaou@wavelet.el.cycu.edu.tw always an important tool to help doctors make diagnosis decisions because it is noninvasive, whereas a surgical operation is invasive [2]. In recent years, the endoscopy has even become an important specialized area in clinical medicine.

In order to acquire a larger field of view inside a small and narrow digestive tract, an endoscope is usually equipped with a wide-angle lens (fish eye lens). Thus, the acquired image often suffers from some degree of deformation distortion called the fish 
eye effect or barrel effect [3-4], resulting in image enlargement around the middle portion and induced distortion extended outward in radial directions. Fig. 1 shows a typic barrel-type distortion.

In fact, experienced doctors with good clinical training can automatically "conjecture" the true size and position of the objects in their minds by viewing the distorted image $[1,5]$. In order to eliminate this conjecture and obtain related information more directly and conveniently, we attempt to develop a specific calibration algorithm to correct the distortion of endoscope images automatically.

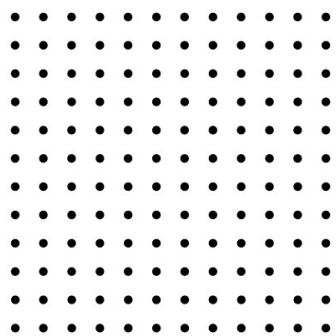

(a)

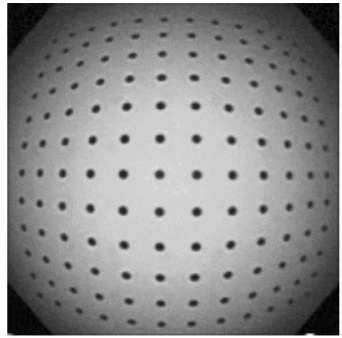

(b)
Fig 1. An example of distorted endoscope image. (a) An original calibration pattern and; (b) The endoscopic distorted image corresponding to (a).

One traditional method for distortion calibration is to draw scales or grid lines on a lens or on a screen directly, and estimate the size of each object in the image by human eyes [6-7]. This kind of method induces a relatively large estimation error. Another intuitive method is the use of a reference image [8]. A referenced endoscope image having known object size and other important information is compared against a distorted target image, so that we can get the relationship between them to estimate the actual object size and retrive other information from the target image. In the same imaging conditions (such as acquired angle, acquired distance, etc.), this kind of method will have high accuracy of estimation, but the accuracy may be lost significantly if those ideal conditions are not met. There is still one more method to measure the size of symptom area, that is, using the stereoscopic endoscope [9-10]. The stereoscopic endoscope can display a three-dimensional stereo image because it has double lens. This kind of method can have an accurate result in general. However, it is not widely used because it is still too expensive to general public.

In recent years, the study of using a calibration pattern to correct the distortion in endoscope images has attracted a lot of attention. In this approach, the first step is to make an uniform dot calibration pattern, followed by using the endoscope to capture the image of the pattern. In 1992, Smith et al. proposed a method to solve the distortion problem in endoscope images [11]. Their idea is as follows. Since the image captured by using a wide-angle lens has circular symmetry, they transform image data in Cartesian coordinates into the concentric circles in polar coordinates and make use of the image grid dots, surface curvature of lens, distance between dots and so on to calculate the symptom area in the acquired image. Using a suitable calibration pattern to correct endoscope images is a low-cost approach but with a reasonable degree of accuracy. Therefore, it deserves an in-depth study.

According to the method proposed by Smith et al. [11], Stefansic et al. proposed a second-order correction method based on a mathematic model [12]. By calculating the expansion coefficients $a_{j}$ in orthogonal Chebyshev polynomials, as shown in Equations (1) and (2), required parameters of the mathematic model are estimated for the distortion correction of endoscope images. The expansion coefficients $a_{i}$ and orthogonal Chebyshev polynomials $P_{j ; N}(i)$ are defined as follows:

$$
\begin{gathered}
a_{j}=\frac{\sum_{i=0}^{N} r_{i}^{\prime} P_{j ; N}(i)}{\sum_{i=0}^{N}\left[P_{j ; N}(i)\right]^{2}}, \\
P_{j ; N}(i)=\sum_{k=0}^{j}(-1)^{k}\left(\begin{array}{c}
j \\
k
\end{array}\right)\left(\begin{array}{c}
j+k \\
k
\end{array}\right) \frac{i^{(k)}}{N^{(k)}},
\end{gathered}
$$

where $r_{i}^{\prime}$ is the distance from the $i$ th point to the image center, $j$ is the order of orthogonal Chebyshev polynomials, $N$ is the number of dots in the calibration pattern, $i^{(k)}$ and $N^{(k)}$ are the $k$ th factorials, or equivalently, $i^{(k)}=i(i-1)(i-2) \ldots(i-k+1), N^{(k)}=N(N-1)(N-$ 2) ...(N-k+1) .

In addition, Tsai also uses the distortion model that is radially symmetrical from the image center to make distortion correction for two dimensional images. His mathematic model is as follows [13]:

$$
\left\{\begin{array}{l}
X_{u}=X_{d}+\left(X_{d}-C_{x}\right)\left(K R_{d}^{2}\right) \\
Y_{u}=Y_{d}+\left(Y_{d}-C_{y}\right)\left(K R_{d}^{2}\right),
\end{array}\right.
$$

where $X_{u}$ and $Y_{u}$ are the coordinates of a corrected image, $X_{d}$ and $Y_{d}$ are the coordinates of a distorted image, $C_{x}$ and $C_{y}$ are the $x$-coordinate and $y$-coordinate of the center of the distorted image, respectively, $R_{d}$ is the distance between an arbitrary point and the center of the distorted image, $K$ is an external camera parameter. The purpose of this model is to translate the coordinates of a distorted image to the coordinates that take the camera lens's center as the origin of the 
coordinates.

Helferty et al. use a non-linear and iterated correction method to calculate the corrected calibration pattern image [14], as shown in Fig. 2. By using the information of dot coordinates of a corrected image, they calculate correction parameters and the correction errors in both horizontal and vertical directions. The correction process is completed when the errors are smaller than a predetermined threshold.

Although using the method of calibration pattern alone may obtain a relatively good result, the specific optical parameters of endoscopic lens, such as radius, angle of view, and focal length, are not considered. Thus, we can not make use of a correction method that is based on a mathematic model.

Haneish at al. proposed a mathematic model to correct image distortion based on the premise that the image is circularly symmetrical about the center [15]. In their approach, the first step is to transform the Cartesian coordinates to the polar coordinates, then calculate the radius $r^{\prime}$ (defined as the distance from the center $\left(x_{c}^{\prime}, y_{c}^{\prime}\right)$ to an arbitrary point in the distorted image) and another radius $r$ (defined as the distance from the image center to the corresponding point) in the original calibration pattern. Specifically, they assume the radius transformation model as follows:

$$
\begin{aligned}
& r^{\prime}=r+a_{1} r^{2}+a_{2} r^{3}+\cdots+a_{n} r^{n+1} \\
& r^{\prime}=\sqrt{\left(x^{\prime}-x_{c}^{\prime}\right)^{2}+\left(y^{\prime}-y_{c}^{\prime}\right)^{2}},
\end{aligned}
$$

where $\left(x^{\prime}, y^{\prime}\right)$ represents the coordinates of the arbitrary point, and $a_{j}$ denotes a coefficient, $j=1,2, \ldots, n$ .The coordinate information of dots is used to find the coefficients in Equation (4), resulting in the correction model of interest. Considering the computational complexity and the correction result, most people adopt the second order $(n=2)$ or the third order $(n=3)$ mathematic model.

In this paper we will use a circular symmetrical model that is similar to that in [15], but noting that the true image central point is normally not on the image center. Therefore, we will modify Equation (4) to reflect this observation. In addition, we propose a simple solution to find a set of suitable parameters for the model.

In the next section, we will introduce the working principle and experimental methods for the proposed approach, followed by experimental results and the conclusion.

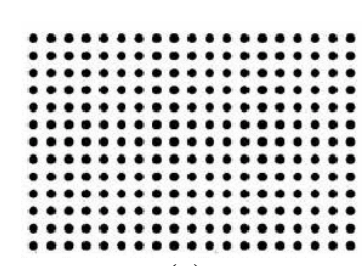

(a)

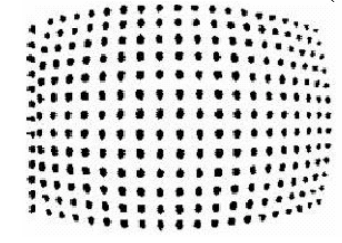

(b)

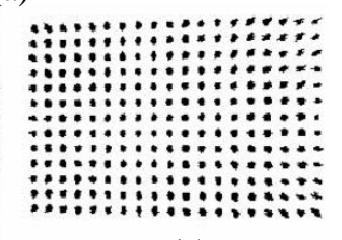

(c)
Fig 2. A calibration pattern. (a) Original calibration pattern; (b) The image of calibration pattern captured by an endoscope; (c) A distortioncorrected image of the image in (b).

\section{THEORY AND METHOD}

\subsection{The Use of Image Processing Techniques}

To measure how serious the endoscopic distortion is, we extract the dots of the calibration pattern and compare them with those in the original calibration pattern to evaluate and quantify the seriousness of distortion. The dots in the calibration pattern are extracted by the use of digital image processing techniques [16-18], and the flowchart of the process is shown in Fig. 3.

After capturing the image of calibration pattern by an endoscope, we perform gray scale processing and reserve only the brightness information of the image. Then an averaging process is adopted to make the gray level of each pixel to be centralized near the average brightness of the image, followed by high pass filtering to emphasize the high frequency component. Next a threshold is selected in the binarization operation to separate dots of calibration pattern from the image background. As for the four corners that are unique in the endoscope image, we create a white triangular object with fixed size and shape to cover them. Then we use a median filter to clean up some noises, leaving only the dots of the calibration pattern. With low pass filtering, we obtain dots having comparatively smoother boundaries. The gravity of each dot is calculated and served as the center of the dot in the calibration pattern and its coordinates are recorded accordingly.

\subsection{The Experimental Design}

\section{The experiment flowchart}


The flowchart of correction experiment for the endoscope image is presented in Fig. 4. The first step is to produce a suitable calibration pattern. Then take the image of calibration pattern by an endoscope and extract the dots of calibration pattern using the technique of digital image processing. Next we find each dot location and the resulting coordinates of each dot are recorded. The parameters of our mathematic model are calculated according to the coordinate information of dots. Then, we transform the coordinates of distorted image into the coordinates of corrected image and carry out the pixel interpolation whenever needed. Finally, we propose an evaluation process to evaluate the correction result.

\section{The coordinates of dot center in calibration pattern}

In Section 2.1, after extracting each dot from the endoscope image, we calculate the coordinates of the dot center and the results for all dots are presented in Table I and Table II, where the coordinates at the upper left corner are $(0,0)$, and the coordinates at the bottom right corner are $(255,255)$.

\section{Second-order circular approximation equation}

Since the endoscope image is taken by the wideangle lens, its image characteristic presents radially symmetrical distortion from the center point. According to [15] and Equation (4), we let

$$
A_{0}+A_{1} r+A_{2} r^{2}=r^{\prime},
$$

where $r^{\prime}$ is the distance between a point and the center in the distorted image of calibration pattern and $r$ is the distance between the corresponding point and the center of the calibration pattern. In Equation (4), the constant coefficient $A_{0}$ is missing, for it was assumed that the image center point will be exactly at the center of the image. In other words, a shift-offset of zero was assumed when taking the image. However, after observing our experiment result, we found that the shift-offset is nonzero, as shown in Fig. 5, particularly Fig. 5(c). Therefore, we add a constant term $A_{0}$ in Equation (4). Further justification and analysis is given below.

For a digital image, every pixel has a pair of integer coordinates. Thus, theoretically, the center of the original calibration pattern should be located at $(128,128)$ for a $256 \times 256$ image. However, in reality, the center is shifted. We use Table I and Table II to illustrate this effect by considering the four innermost points around the center of calibration pattern. They are $(117,119),(137,119),(117,139)$, and $(137,139)$ in Table I. By taking the average of these four pairs of coordinates and rounding to the nearest integer, we obtain the center coordinates $(127,129)$ of the original image. Similarly, taking the four central points, (117, 103), (146, 103), (117, 131), and $(146,131)$ in Table

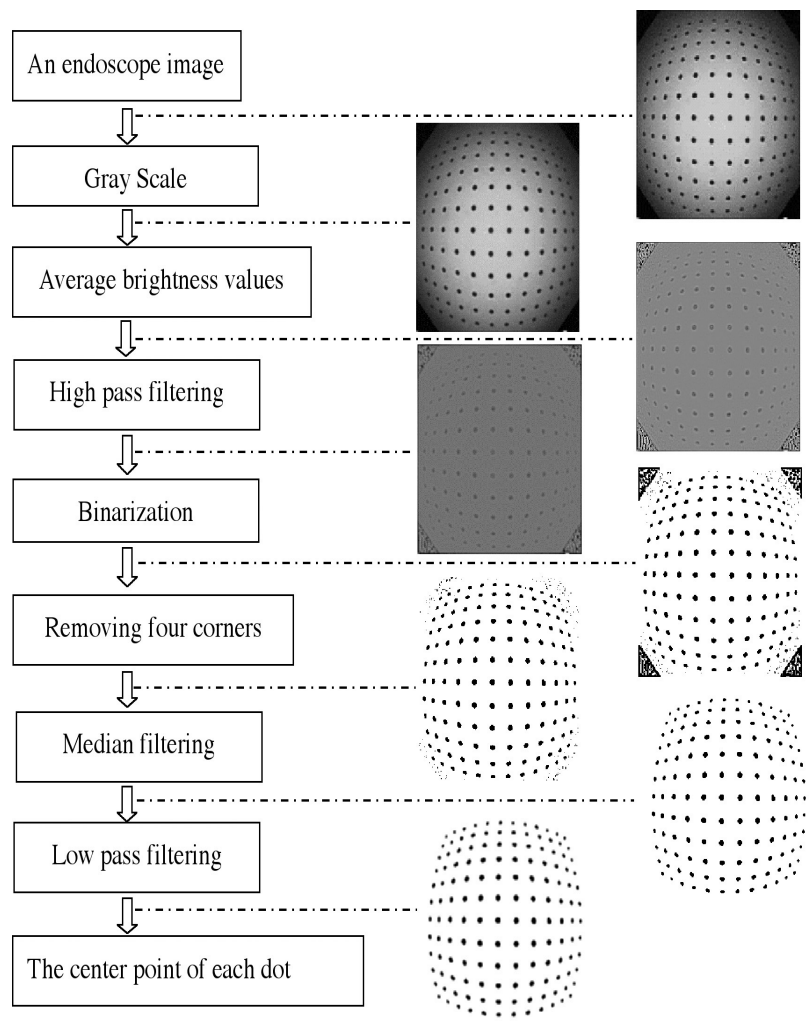

Fig 3. The flowchart of extracting dots in an image with calibration pattern.

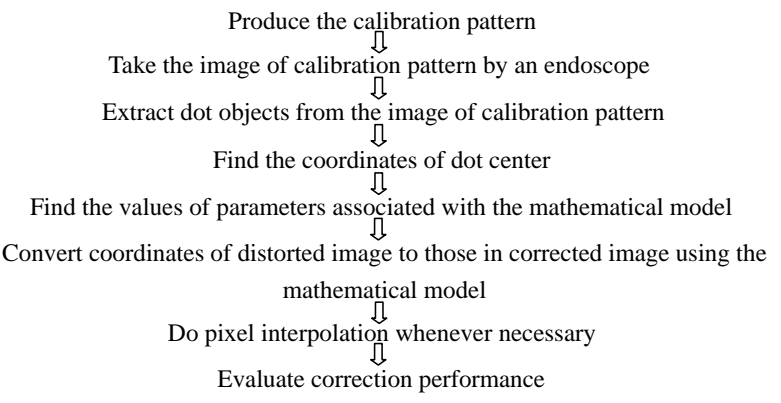

Fig 4. The flowchart of correction experiment.

Table I. The coordinates of dot center in original image of calibration pattern.

\begin{tabular}{|c|c|c|c|c|c|c|c|c|c|c|c|}
\hline ,18 & 36,18 & 56,18 & 76,18 & 97,18 & 117,18 & 137,18 & 157,18 & 177,18 & 198,18 & 218,18 & 238,18 \\
\hline 5,38 & 36,38 & 56,38 & 6,38 & 97,38 & 117,38 & 137,38 & 157,38 & 177,38 & 198,38 & 218,38 & 238,38 \\
\hline 15,59 & 36,58 & 56,58 & 76,58 & 97,59 & 117,58 & 137,58 & 157,59 & \begin{tabular}{|l|l}
177,59 \\
\end{tabular} & \begin{tabular}{|l|l|}
198,58 \\
\end{tabular} & 218,58 & 238,58 \\
\hline 5,79 & 36,79 & 56,79 & 76,79 & 97,79 & 117,78 & 137,78 & 157,79 & 177,79 & 198,79 & 218,79 & 238,79 \\
\hline 5,99 & 36,99 & 56,99 & 76,99 & 97,99 & 117,99 & 137,99 & 157,99 & 177,99 & 198,99 & 218,99 & 238,99 \\
\hline 5,119 & 936,119 & 56,119 & 76,119 & 97,11 & 117,1 & 137,11 & 157,11 & 1177,1 & 98,1 & 218,1 & 9238,119 \\
\hline 5,13 & 936,139 & $956,13$. & 76,13 & $\begin{array}{ll}91,1 \\
.\end{array}$ & 117 & 137,1 & 157,1 & 177 & 198,1 & & 38,139 \\
\hline 16 & & 0, & 0,1 & I, & & & & 17 & & & 8,159 \\
\hline 18 & & & & 7.18 & & 137,17 & & & & & 238,180 \\
\hline 15,200 & 036,200 & 56,2 & 6,17 & 97,20 & & 137 & 157, & 9177,1 & 98,17 & 218,1 & 238,179 \\
\hline 15,22 & 036,220 & 56,220 & 76,220 & 97,22 & 11722 & 137,22 & 157,22 & 0177, & 198,2 & 218,2 & 238,220 \\
\hline & & & & & & & & & & & 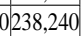 \\
\hline
\end{tabular}


Table $\Pi$. The coordinates of dot center in the image of distorted calibration pattern.

\begin{tabular}{|l|l|l|l|l|l|l|l|l|l|l|l|}
\hline 36,28 & 49,23 & 64,19 & 82,15 & 101,12 & 121,11 & 141,12 & 160,13 & 178,16 & 195,21 & 210,25 & 222,30 \\
\hline 31,40 & 45,35 & 60,30 & 78,27 & 99,25 & 120,23 & 142,23 & 162,25 & 182,28 & 200,32 & 215,37 & 228,43 \\
\hline 25,54 & 39,50 & 56,45 & 75,42 & 96,39 & 119,39 & 143,38 & 165,40 & 186,43 & 204,47 & 220,51 & 233,57 \\
\hline 21,70 & 34,66 & 51,62 & 71,59 & 94,57 & 119,57 & 144,56 & 168,58 & 190,61 & 209,64 & 225,68 & 238,73 \\
\hline 16,88 & 30,85 & 47,83 & 68,80 & 92,78 & 118,78 & 145,78 & 170,79 & 193,82 & 213,85 & 229,88 & 242,91 \\
\hline 13,108 & 27,107 & 44,105 & 65,104 & 90,103 & $\mathbf{1 1 7 , 1 0 3}$ & $\mathbf{1 4 6 , 1 0 3}$ & 172,104 & 196,106 & 216,107 & 232,109 & 245,110 \\
\hline 11,129 & 25,130 & 42,130 & 63,130 & 89,130 & $\mathbf{1 1 7 , 1 3 1}$ & $\mathbf{1 4 6 , 1 3 1}$ & 173,131 & 197,131 & 217,132 & 234,131 & 247,132 \\
\hline 10,151 & 24,153 & 42,155 & 63,157 & 89,159 & 117,160 & 146,160 & 173,159 & 197,158 & 217,156 & 234,154 & 246,152 \\
\hline 12,171 & 26,175 & 43,179 & 65,182 & 89,185 & 117,186 & 145,186 & 172,185 & 195,183 & 215,180 & 231,176 & 245,173 \\
\hline 15,191 & 29,196 & 46,201 & 67,205 & 91,209 & 118,211 & 145,210 & 170,208 & 193,205 & 212,201 & $228,196241,191$ \\
\hline 20,207 & $33,21451,219$ & 70,225 & 93,228 & 118,230 & 143,230 & 167,228 & 189,224 & 207,219 & 224,214 & 237,208 \\
\hline $25,22439,230$ & 55,236 & 74,241 & 95,244 & 118,246 & 142,246 & 164,244 & 185,240 & 203,234 & 218,229 & 231,223 \\
\hline
\end{tabular}

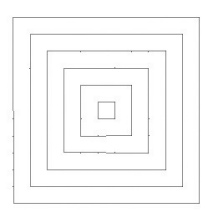

(a)

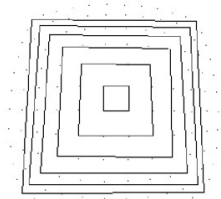

(b)

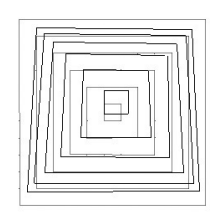

(c)
Fig 5. The quadrilateral linearity test for calibration patterns. (a) The original calibration pattern; (b) The distorted calibration pattern; (c) The result of superimposing the original image and corresponding distorted image of calibration pattern.

II, we get the center coordinates $(132,117)$ of the distorted image. This shows the non-zero shift of the image center.

Again, Fig. 5(c) clearly shows the shift of the center of distorted pattern image. Therefore, adding the constant term $A_{0}$ to modify Equation (4) is justified.

In the original calibration pattern, we take the points at $(x, y)=(137,119),(157,99),(177,79),(198$, $58),(218,38)$, and $(238,18)$, and consider

$$
r=\sqrt{\left(x-x_{c}\right)^{2}+\left(y-y_{c}\right)^{2}}
$$

and

$$
r^{\prime}=\sqrt{\left(x^{\prime}-x_{c}^{\prime}\right)^{2}+\left(y^{\prime}-y_{c}^{\prime}\right)^{2}},
$$

where $(x, y)$ is the coordinate pair for a point in the original pattern image, $\left(x_{c}, y_{c}\right)$ is the central coordinate pair of the original pattern image, and $r$ is the radius of circles centered at $\left(x_{c}, y_{c}\right)$. By Equation (7), we obtain $r=14.5,42.43,70.71,100.41,128.7$, and 157.33, respectively. Similarly, by Equation (8), we can get six corresponding points of distorted pattern image from Table $I$ and result in six radii of circles centered at $\left(x_{c}{ }^{\prime}, y_{c}{ }^{\prime}\right)$ in the distorted image. They are $r^{\prime}=20.16$, $54.1,80.98,100.77,115.6$, and 125.53 respectively. Of course we can also take another set of six corresponding points to compute the parameters of our mathematic model.

We have six known pairs of $\left(r=r^{\prime}\right)$ in Equation (6) but only have three unknown parameters or coefficients $A_{0}, A_{1}$, and $A_{2}$. Hence we consider only three pairs of $\left(r=r^{\prime}\right)$, say $r=14.5,70.71,157.33$ and $r^{\prime}=20.16,80.98,125.53$, in Equation (6) and solve for these simultaneous equations to get coefficients $A_{0}=0.421, A_{1}=1.408$, and $A_{2}=-3.92 \times 10^{-3}$. Therefore, Equation (6) becomes:

$$
0.421+1.408 r-3.92 \times 10^{-3} r^{2}=r^{\prime}
$$

Now we have established the radius mapping between the dots in the original image and those in the distorted image. So given a radius $r^{\prime}$, we can estimate the corresponding radius $\hat{r}$ as follows:

$$
0.421+1.408 \hat{r}-3.92 \times 10^{-3}(\hat{r})^{2}=r^{\prime},
$$

where $\hat{r}$ is the radius from a point to the center in a corrected image.

Fig. 6 shows the flowchart of coordinate transformation between the distorted and the corrected images. In Fig. $6,\left(x^{\prime}, y^{\prime}\right)$ is a known coordinate pair in the distorted image. We transform $\left(x^{\prime}, y^{\prime}\right)$ in the Cartesian coordinates to $\left(r^{\prime}, \theta^{\prime}\right)$ in the polar coordinates. By substituting $r^{\prime}$ in Equation (10), we obtain the corrected or estimated radius $\hat{r}$. Let $\hat{\theta}=\theta$. Then the corrected coordinate pair $(\hat{x}, \hat{y})$ corresponding to $\left(x^{\prime}, y^{\prime}\right)$ is obtained. Finally, the pixel value at $\left(x^{\prime}, y^{\prime}\right.$ ) is assigned to that at $(\hat{x}, \hat{y})$. However, for the pixels that were not mapped from $\left(x^{\prime}, y^{\prime}\right)$, they are filled by the values obtained with bilinear interpolation. In other words, the pixel value of concern is obtained by combining the contribution from its four surrounding pixels.

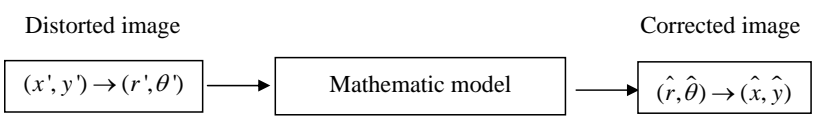

Fig 6. The flowchart of coordinate transformation.

According to Equation (6), only three unknown coefficient $A_{0}, A_{1}$, and $A_{2}$ are to be determined. But we have six pairs of $\left(r, r^{\prime}\right)$. In fact, any of three pairs are possible to obtain a solution and there could be at least $\left(\begin{array}{l}6 \\ 3\end{array}\right)=20$ different solutions. The solution expressed as Equation (10) is only one of the solutions, which could be suboptimal. Sometimes we may choose unsuitable parameters based on a bad solution, so that the correction result is not good enough. One bad example is shown in Fig. 7. A new solution using all six pairs of $\left(r, r^{\prime}\right)$ simultaneously is presented next. 


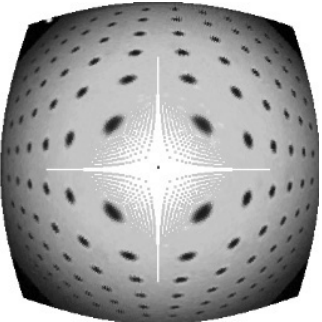

Fig 7. A bad correction result due to the use of unsuitable parameters in our mathematic model.

\section{Least Square Regression}

Instead of finding an exact solution based on three pairs of $\left(r, r^{\prime}\right)$, we intend to find an approximate solution based on all six pairs of $\left(r, r^{\prime}\right)$. In other words, we hope to find the coefficients that can minimize overall errors when all six pairs of $\left(r, r^{\prime}\right)$ are considered in Equation (6). With this idea, we form the matrix equation as follows:

$$
\left[\begin{array}{ccc}
1 & 14.50 & 210.25 \\
1 & 42.43 & 1800 \\
1 & 70.71 & 5000 \\
1 & 100.41 & 10082.17 \\
1 & 128.70 & 16563.69 \\
1 & 157.33 & 24752.73
\end{array}\right]\left[\begin{array}{l}
A_{0} \\
A_{1} \\
A_{2}
\end{array}\right]=\left[\begin{array}{c}
20.16 \\
54.10 \\
80.98 \\
100.77 \\
115.60 \\
125.53
\end{array}\right]
$$

By letting

$$
\mathbf{R}=\left[\begin{array}{ccc}
1 & 14.50 & 210.25 \\
1 & 42.43 & 1800 \\
1 & 70.71 & 5000 \\
1 & 100.41 & 10082.17 \\
1 & 128.70 & 16563.69 \\
1 & 157.33 & 24752.73
\end{array}\right], \quad \mathbf{A}=\left[\begin{array}{c}
A_{0} \\
A_{1} \\
A_{2}
\end{array}\right], \quad \text { and } \quad \mathbf{R}^{\prime}=\left[\begin{array}{c}
20.16 \\
54.10 \\
80.98 \\
100.77 \\
115.60 \\
125.53
\end{array}\right] \text {, }
$$

Equation (11) can be rewritten as $\mathbf{R A}=\mathbf{R}^{\mathbf{}}$. $\mathbf{A}=\mathbf{R}^{\mathbf{1}}$ $\mathbf{R}^{\prime}$ if $\mathbf{R}$ is a nonsingular square matrix. Unfortunately, is not. So we must find a set of optimum solution $\mathbf{A}$ that can minimize the square of the norm of the error vector $\mathbf{e}$ :

$$
\|\mathbf{e}\|^{2}=\left\|\mathbf{R A}-\mathbf{R}^{\prime}\right\|^{2}
$$

This is known as the pseudo inverse solution. Using $\left(\mathbf{R}^{T} \mathbf{R}\right)=\mathbf{R}^{T} \mathbf{R}^{\prime}$ to solve for $\mathbf{A}$, we have

$$
\mathbf{A}=\left[\begin{array}{l}
A_{0} \\
A_{1} \\
A_{2}
\end{array}\right]=\left[\begin{array}{c}
1.7468 \\
1.3728 \\
-0.0037505
\end{array}\right] .
$$

With this solution, Equation (6) can be written as

$$
1.7468+1.3728 r-0.0037505 r^{2}=r^{\prime}
$$

Since the parameters of our mathematic model are obtained, we can correct distorted images according to the experiment methods mentioned above. The evaluation of experiment and correction results are given in the next section.

\section{EXPERIMENTAL RESULT}

\subsection{Correction Result}

We obtain a set of essential coefficients for the correction model by the least square regression method discussed in Section 2.2, and then get the resulting image by correction and interpolation. Fig. 8 shows an actual image of the gastrointestinal tract and Fig. 9 and Fig. 10 show the correction results of the calibration pattern and the actual image of gastrointestinal tract, respectively.

In Fig. 9, we could find the phenomenon of rippling around the image. That is because we have used a second-order mathematical model (in fact, it is a model of a concentric circles). For corrected images, we cut off the part we need. In the case of Fig. 9, we cut off all dots on the calibration pattern in order to estimate the correction result, resulting in Fig. 11. The same square size as in Fig. 11 is applied to Fig. 10 to obtain Fig. 12.

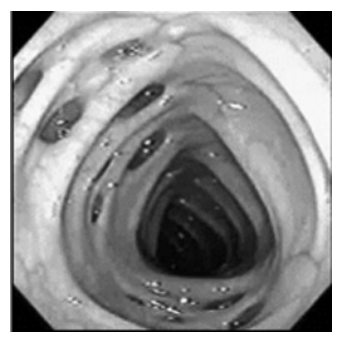

Fig 8. An actual image of the gastrointestinal tract.

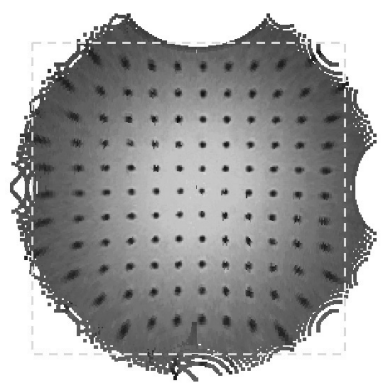

Fig 9. A correction result for the calibration pattern. 


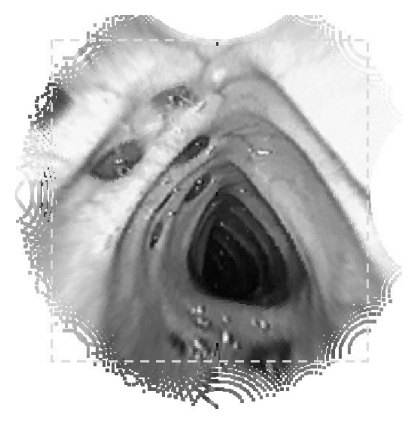

Fig 10. A correction result for the actual image of the gastrointestinal tract given in Fig. 8.

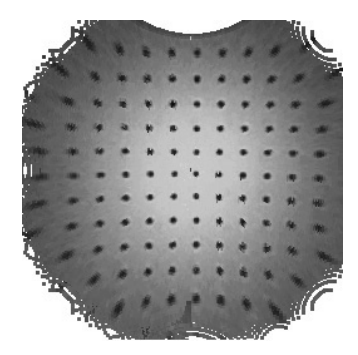

Fig 11. A square cut of Fig. 9.

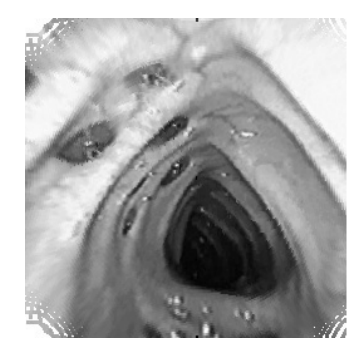

Fig 12. A square cut of Fig. 10.

We can find that a corrected image is slightly larger than its original one, and this is due to the magnification effect of the optical lens. For a traditional endoscope, the magnification of the lens is 1.61. So we can estimate the image size after correction from the original one. It has no effect on the correction results because the difference between original and corrected images is within a scale factor. Hence, given a certain scalar we are able to reduce the image size by the bilinear interpolation, such that the sizes of the corrected and the original images are the same.

Using the dot extraction method based on digital image processing techniques, as discussed in Section 2.1, dots on the calibration pattern in Fig. 11 can be extracted. Then we find the coordinates of each dot center by finding the gravity of dot. The coordinates of the dots in Fig. 11 are marked in Table III. A few dots are marked with an " $\mathrm{x}$ ". This means that some dots are not restored. This result matches that in Fig. 11, where we can easily see that some dots in the calibration pattern do disappear.

Table III. The coordinates of the dots in the corrected image of the calibration pattern.

\begin{tabular}{|c|c|c|c|c|c|c|c|c|c|c|c|}
\hline $\mathrm{x}$ & 33,20 & 54,21 & 76,23 & 98,26 & 118,25 & 138,25 & 158,25 & 179,25 & 200,26 & 223,22 & $\mathrm{x}$ \\
\hline $\mathrm{x}$ & 34,42 & 58,46 & 78,46 & 99,46 & 120,46 & 138,46 & 158,46 & 178,46 & 198,47 & 220,46 & 245,44 \\
\hline 8,61 & 37,65 & 57,64 & 80,65 & 100,65 & 120,65 & 138,66 & 158,66 & 177,67 & 198,65 & 218,67 & 240,66 \\
\hline 11,83 & 38,85 & 60,85 & 81,85 & 101,86 & 120,86 & 138,86 & 157,86 & 177,85 & 197,85 & 216,86 & 237,86 \\
\hline 16,105 & 39,104 & 61,104 & 82,104 & 101,104 & 120,104 & 138,104 & 157,104 & 176,105 & 195,105 & 215,106 & 236,106 \\
\hline 16,124 & 40,123 & 62,123 & 82,123 & 102,122 & $\mathbf{1 2 1 , 1 2 2}$ & $\mathbf{1 3 8 , 1 2 3}$ & 156,123 & 175,123 & 195,124 & 215,125 & 235,125 \\
\hline 13,146 & 41,144 & 61,142 & 82,141 & 101,141 & $\mathbf{1 2 0 , 1 4 0}$ & $\mathbf{1 3 8 , 1 4 0}$ & 156,141 & 175,142 & 195,143 & 214,144 & 235,146 \\
\hline 7,169 & 42,165 & 61,162 & 81,161 & 101,160 & 120,159 & 138,159 & 157,160 & 175,161 & 195,161 & 214,163 & 237,166 \\
\hline $\mathrm{x}$ & 35,187 & 61,182 & 81,181 & 100,180 & 119,179 & 138,179 & 157,179 & 175,180 & 194,182 & 215,184 & 238,187 \\
\hline $\mathrm{x}$ & $\mathrm{x}$ & 56,207 & 79,203 & 100,200 & 119,200 & 138,199 & 157,199 & 176,201 & 195,202 & 216,205 & $\mathrm{x}$ \\
\hline $\mathrm{x}$ & $\mathrm{x}$ & $\mathrm{x}$ & 77,228 & 97,226 & 118,223 & 138,223 & 158,223 & 178,227 & 198,232 & $\mathrm{x}$ & $\mathrm{x}$ \\
\hline $\mathrm{x}$ & $\mathrm{x}$ & $\mathrm{x}$ & $\mathrm{x}$ & $\mathrm{x}$ & $\mathrm{x}$ & $\mathrm{x}$ & $\mathrm{x}$ & $\mathrm{x}$ & $\mathrm{x}$ & $\mathrm{x}$ & $\mathrm{x}$ \\
\hline
\end{tabular}

\subsection{Evaluation Criteria}

After reviewing the literature related to this study, we found no standard criterion to evaluate the correction performance. The reasons for this are as follows:

(1) The difference in calibration patterns - there is no unified specification on the distance between adjacent dots or the size and shape of dots.

(2) The use of different endoscopic instruments the viewing angle and the resolution of lens, as well as the size of acquired images are different.

(3) Normally, no corresponding image of gastrointestinal tract taken by a normal (not wideangle) lens is available.

In addition, according to the optical characteristic, the focal length of endoscope will affect the viewing angle, resulting in different degrees of distortion. Furthermore, the size or shape (such as square or rectangular) of acquired images will make a difference in the manufacture of calibration pattern.

According to the discussion above, it is difficult to evaluate the correction performance. Hence we consider several evaluation methods as follows:

(1) Perform a subjective test by viewing the images directly. This part must be judged by professional physicians.

(2) Perform an objective test by comparing the corrected image of calibration pattern with the original one. This is done by calculating the correction errors in both horizontal and vertical directions based on the coordinates of dot centers.

Since we are not physicians, here we discuss the objective test only. 
Table IV. The coordinates of dot centers in corrected image from Table III.

\begin{tabular}{|c|c|c|c|c|c|c|c|c|c|c|c|}
\hline $\mathrm{x}$ & 31,18 & 52,19 & 74,21 & 96,24 & 116,23 & 136,23 & 156,23 & 177,23 & 198,24 & 221,20 & $\mathrm{x}$ \\
\hline $\mathrm{x}$ & 32,40 & 56,44 & 76,44 & 97,44 & 118,44 & 136,44 & 156,44 & 176,44 & 196,45 & 218,44 & 243,42 \\
\hline 6,59 & 35,63 & 55,62 & 78,63 & 98,63 & 118,63 & 136,64 & 156,64 & 175,65 & 196,63 & 216,65 & 238,64 \\
\hline 9,81 & 36,83 & 58,83 & 79,83 & 99,84 & 118,84 & 136,84 & 155,85 & 175,83 & 195,83 & 214,84 & 235,84 \\
\hline 14,103 & 37,102 & 59,102 & 80,102 & 99,103 & 118,102 & 136,102 & 155,102 & 174,103 & 193,103 & 213,104 & 234,104 \\
\hline 14,122 & 38,121 & 60,121 & 80,121 & 100,120 & 119,120 & 136,121 & 154,121 & 173,121 & 193,122 & 215,123 & 233,123 \\
\hline 11,144 & 39,142 & 59,140 & 80,139 & 99,139 & 118,138 & 136,138 & 154,139 & 173,140 & 193,141 & 212,142 & 233,144 \\
\hline 5,167 & 40,163 & 59,160 & 79,159 & 99,158 & 118,157 & 136,157 & 155,158 & 173,159 & 193,159 & 212,161 & 235,164 \\
\hline $\mathrm{x}$ & 33,185 & 59,180 & 79,179 & 98,178 & 117,177 & 136,176 & 155,177 & 173,178 & 195,180 & 213,182 & 236,185 \\
\hline $\mathrm{x}$ & $\mathrm{x}$ & 54,205 & 77,201 & 98,198 & 117,198 & 136,197 & 155,197 & 174,199 & 193,200 & 214,203 & $\mathrm{x}$ \\
\hline $\mathrm{x}$ & $\mathrm{x}$ & $\mathrm{x}$ & 75,226 & 95,224 & 116,221 & 136,221 & 156,221 & 176,225 & 196,230 & $\mathrm{x}$ & $\mathrm{x}$ \\
\hline $\mathrm{x}$ & $\mathrm{x}$ & $\mathrm{x}$ & $\mathrm{x}$ & $\mathrm{x}$ & $\mathrm{x}$ & $\mathrm{x}$ & $\mathrm{x}$ & $\mathrm{x}$ & $\mathrm{x}$ & $\mathrm{x}$ & $\mathrm{x}$ \\
\hline
\end{tabular}

We calculate the average location of the four innermost dots located at $(121,122),(138,123),(120$, $140)$, and $(138,140)$, respectively, in Table III and obtain the integer central point $(129,131)$ after performing a rounding off operation. The coordinates of this central point are compared with those in the original calibration pattern or $(127,129)$. Obviously we have a two-pixel shift downward and to the right, respectively. Therefore, we subtract the $(\mathrm{X}, \mathrm{Y})$ coordinates of each dot center from Table III and obtain the corresponding result in Table IV.

In general, images can only be evaluated by comparing an original image of calibration pattern with its distorted and corrected version because we have reference points (the dots of calibration pattern) to make a comparison. Therefore, we do not use the entire corrected image (like the one in Fig. 11) to evaluate, instead we examine the coordinates of the dots of the calibration pattern.

We list the average errors in both horizontal and vertical directions of dot centers that are in the original image of calibration pattern, in the acquired endoscope image of the pattern, and in the corrected one. First we list the average coordinate values of dot centers in the $\mathrm{X}$ and $\mathrm{Y}$ directions for the $12 \times 12$ dots in the calibration pattern. Next we define the following error:

Error $1=\mid$ the dot coordinate of actual pattern-the dot coordinate of distorted pattern $\mid$ (15a)

Error $2=\mid$ the dot coordinate of actual pattern-the dot coordinate of corrected pattern $\mid(15 b)$

The experimental results are given in Table $\mathrm{V}$ and Table VI for the $\mathrm{X}$ coordinate and the Y coordinate, respectively.

Table V. The comparison of the average $X$ coordinate of dot center.

\begin{tabular}{ll|l|l|l|l|l|l|l|l|l|l|l|l|l} 
coordinate & Col.1 & Col.2 & Col.3 & Col.4 & Col.5 & Col.6 & Col.7 & Col.8 & Col.9 & Col.10 & Col.11 & Col.12 \\
\hline
\end{tabular} \begin{tabular}{|l|l|l|l|l|l|l|l|l|l|l|l|l|l|}
\hline \hline Original image & 15.00 & 36.00 & 56.00 & 76.00 & 97.00 & 117.00 & 137.00 & 157.00 & 177.00 & 198.00 & 218.00 & 238.00 \\
\hline
\end{tabular}

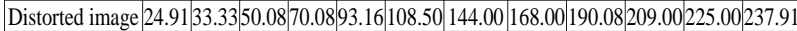
\begin{tabular}{|l|l|l|l|l|l|l|l|l|l|l|l|l|}
\hline Corrected image & 9.83 & 35.66 & 57.10 & 77.90 & 98.00 & 117.54 & 136.00 & 155.18 & 174.45 & 194.63 & 214.80 & 235.87 \\
\hline
\end{tabular} \begin{tabular}{|l|l|l|l|l|l|l|l|l|l|l|l|l|} 
Error 1 & 9.91 & 2.67 & 5.92 & 5.92 & 3.84 & 8.50 & 7.00 & 11.00 & 13.08 & 9.00 & 7.00 & 0.09 \\
\hline
\end{tabular} \begin{tabular}{l|l|l|l|l|l|l|l|l|l|l|l|l|} 
Error 2 & 5.17 & 0.44 & 1.10 & 1.90 & 1.00 & 0.54 & 1.00 & 1.82 & 2.55 & 3.27 & 3.20 & 2.13 \\
\hline
\end{tabular}
Table VI. The comparison of the average of $Y$ coordinates of dot centers.

\begin{tabular}{|c|c|c|c|c|c|c|c|c|c|c|c|c|}
\hline $\begin{array}{c}\text { Y } \\
\text { Coordinate }\end{array}$ & $\begin{array}{c}\text { Row } \\
1\end{array}$ & $\begin{array}{c}\text { Row } \\
2\end{array}$ & $\begin{array}{c}\text { Row } \\
3\end{array}$ & $\begin{array}{c}\text { Row } \\
4\end{array}$ & $\begin{array}{c}\text { Row } \\
5\end{array}$ & $\begin{array}{c}\text { Row } \\
6\end{array}$ & $\begin{array}{c}\text { Row } \\
7\end{array}$ & $\begin{array}{c}\text { Row } \\
8\end{array}$ & $\begin{array}{c}\text { Row } \\
9\end{array}$ & $\begin{array}{c}\text { Row } \\
10\end{array}$ & $\begin{array}{c}\text { Row } \\
11\end{array}$ & $\begin{array}{c}\text { Row } \\
12\end{array}$ \\
\hline $\begin{array}{c}\text { Original } \\
\text { image }\end{array}$ & 18.00 & 38.00 & 58.33 & 78.83 & 99.00 & 119.00 & 139.00 & 159.16 & 179.58 & 199.33 & 220.00 & 240.00 \\
\hline $\begin{array}{c}\text { Distorted } \\
\text { Image }\end{array}$ & 18.75 & 30.66 & 45.41 & 62.58 & 82.91 & 105.75 & 130.66 & 156.16 & 180.08 & 202.00 & 220.50 & 236.41 \\
\hline $\begin{array}{c}\text { Corrected } \\
\text { image }\end{array}$ & 21.80 & 43.54 & 63.16 & 83.41 & 102.66 & 121.33 & 140.5 & 160.16 & 179.72 & 199.77 & 224.00 & $x$ \\
\hline Error 1 & 0.75 & 7.34 & 12.92 & 16.25 & 16.09 & 13.25 & 8.34 & 3.00 & 0.50 & 2.67 & 0.50 & 3.59 \\
\hline Error 2 & 3.80 & 5.54 & 4.83 & 4.58 & 3.66 & 2.33 & 1.50 & 1.00 & 0.24 & 0.44 & 4.00 & $x$ \\
\hline
\end{tabular}

Error 1 reflects the degree of the deviation apart from the undistorted one that a distorted image can have. A shift as great as 16 pixels is observed in Table VI. This result coincides with the characteristic of endoscope image, that is, the central part is magnified. Error 2 reflects the performance of correction. In both Table V and Table VI, Error 2 is obviously smaller than Error 1 (except Col. 12, Row 1, and Row 11), which verifies the validity of correction.

According to the result of Error 2, the error is relatively small in the central part of corrected image, and the error is slightly larger near the boundary of the image. That coincides with the result of our direct observation. The errors in some parts are even less than one pixel and this shows how effective our correction method is.

We have observed and evaluated the relative positions of dot centers. However, one of the main applications of endoscope images is to measure the size of a nodus area. Hence we discuss the accuracy of area measurement further. Given the $12 \times 12$ calibration pattern, we can draw six circumscribed rectangles, and calculate the area enclosed by four corresponding adjacent dots as shown in Fig. 13, 14, and 15. Next we definite the following correction errors:

$$
\text { Area error } 1=\frac{\mid \text { area of original image }- \text { area of distorted image } \mid}{\text { area of ideal image }} \times 100 \%
$$

Area error $2=\frac{\mid \text { area of original image }- \text { area of corrected image } \mid}{\text { area of ideal image }} \times 100 \%$

The results are summarized in Table VII.

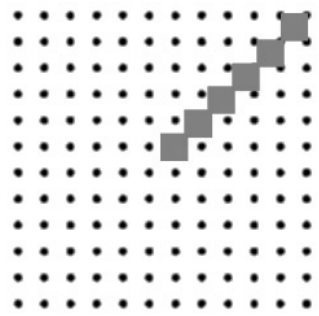

Fig 13. The six areas of original calibration pattern. 


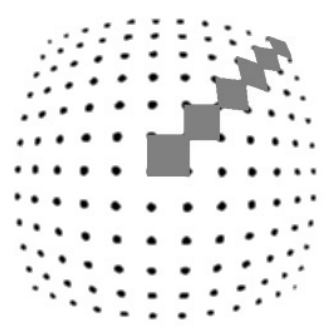

Fig 14. The six areas of distorted calibration pattern.

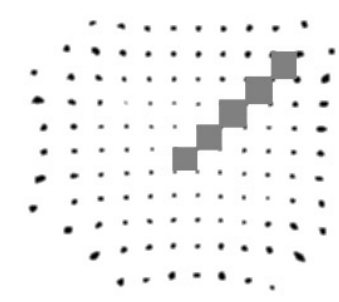

Fig 15. The six areas of corrected calibration pattern.

Table VII. The area comparison for the calibration patterns (unit: number of pixels).

\begin{tabular}{|c|c|c|c|c|c|c|c|c|}
\hline & Area 1 & Area 2 & Area 3 & Area 4 & Area 5 & Area 6 & Average \\
\hline Ideal image & 400 & 400 & 400 & 400 & 400 & 400 & $\mathrm{x}$ \\
\hline Original image & 400 & 400 & 400 & 441 & 400 & 400 & $\mathrm{x}$ \\
\hline Distorted image & 812 & 650 & 672 & 324 & 225 & 156 & $\mathrm{x}$ \\
\hline Corrected image & 394 & 352 & 370.5 & 400 & 389.5 & $\mathrm{x}$ & $\mathrm{x}$ \\
\hline \hline Area Error 1 & $103 \%$ & $62.5 \%$ & $93 \%$ & $19 \%$ & $43.8 \%$ & $61 \%$ & $76.46 \%$ \\
\hline Area Error 2 & $1.5 \%$ & $12 \%$ & $7.3 \%$ & $0 \%$ & $2.6 \%$ & $\mathrm{x}$ & $4.68 \%$ \\
\hline
\end{tabular}

The distance between adjacent dots in an idea image of calibration pattern is fixed to be 20 and thus the area 400 in Table VII is also fixed. Area 4 of actual image is 441 or $21 \times 21$ rather than 400 because some inaccuracy was induced when we extract the dot center by the technique of digital image processing and when we round off the coordinates of the dot to an integer. Thus, the error induced by computer processing exists. However, it is not serious.

According to Table VII, the average error after correction is $4.68 \%$, whereas the average error of distorted image is $76.46 \%$. Hence we can observe that the distortion effect is reduced significantly. When the area error is within an acceptable range, the accuracy of measuring a nodus area will also be improved, and it is useful for physicians to make clinical diagnosis.

\section{CONCLUSION}

Because of the optical physics of a wide-angle lens, the acquired endoscope image could be seriously distorted. We propose a method to correct the distortion. First, we make use of digital image processing techniques, such as binarization, median filter, high pass filter, etc., to extract the dots of calibration pattern from the image. Next we propose a concentric circular model based on a second-order approximation equation, and use the dots of calibration pattern to find the image center. A coordinate transform between Cartesian and polar coordinates is performed to facilitate the finding of the corresponding relationship between the distorted image and the corrected image. We only need to find an antideformation mathematic model for a particular endoscopic lens once, and we can use the model directly to correct all acquired images as long as they are taken by the endoscope with that lens inside.

The deviation of dot pattern between the corrected image and the original image is evaluated. It is expressed by the error or position shift in both the horizontal and the vertical directions as well as the area enclosed by four adjacent dots. The experimental result shows excellent correction performance. We may use a higher order calibration model to obtain more accurate results. However, the computational complexity will also be increased accordingly. In summary, the proposed method is a low-cost approach with excellent correction performance.

\section{ACKNOWLEDGEMENT}

A special thank goes to Professor Yung-Nien Sun for providing Fig. 1 of this paper.

\section{REFERENCE}

1. The Endoscope Development, http://www.dest.org.tw/ icon/icon5-1.html, July 10, 2004.

2. Leggett K: Endoscopy: many pluses, still a few minuses. Biophotonics Int. 1999; 6: 50-53.

3. Castleman KR: Digital Image Processing, Englewood Cliffs, NJ: Prentice-Hall 1996.

4. Kato H and Barron JP: Electronic Videoendoscopy, Switzerland: Harwood 1993.

5. Chien TY: Auto-calibration, reconstruction and assessment of clinical lesions from endoscope image sequence. National Cheng-Kung University, Computer Science \& Information Engineering, Master thesis; 2002. 
6. Murayama M, Ichioka S, Takemoto T, Tsuda K and Hori S: A clinical trial of a gastrofiberscope with scale. Gastroenterol Endoscope 1971; 13: 49-57.

7. Sakita T and Oguro Y: Measurements of the lesion size under direct vision with the optical fiberscope. Gastrointest Endoscope1973; 15: 456-464.

8. Vakil N, Smith WE, Bourgeois K and Maislin SA: Endoscopic measurement of lesion size: improved accuracy with image processing. Gastrointest Endoscope 1992; 40: 178-183.

9. Catalano MF, Van Dam J, Bedford R, Cothren RM and Sivak MV Jr: Preliminary evaluation of the prototype stereoscopic endoscope: precise threedimensional measurement system. Gastrointest Endoscope 1993; 39: 23-28.

10. http://www.epochtimes.com/b5/3/2/10/n274332.htm, July 10, 2004 。

11. Smith WE, Vakil N and Maislin SA: Correction of distortion in endoscope images. IEEE Trans. Medical Imaging 1992; 11: 117-122.

12. Stefansic JD, Herline AJ, Chapman WC and Galloway RL Jr: Endoscopic tracking for use in interative, image-guided surgery. SPIE Med. Imag., Image Display 1998; 3335: 208-215.

13. Tsai RY: An efficient and accurate camera calibration technique for 3D machine vision. Proc. of IEEE Computer Vision and Pattern Recognition 1986; 364-374.

14. Helferty JP, Zhang C, McLennan G and Higgins WE: Videoendoscopic distortion correction and its application to virtual guidance of endoscopy. IEEE Trans. Medical Imaging 2001; 20: 605-617.

15. Haneish H and Miyake Y: Distortion compensation of electronic endoscope image. IEEE Conf. Rec. of Medical Image Conf. 1994; 3: 1717-1721.

16. Lehmann TM, Gonner C and Spitzer K: Survey: interpolation methods in medical image processing. IEEE Trans. Medical Imaging 1999; 18: 10491075.

17. Gonzalez RC and Woods RE: Digital Image Processing, 2nd ed., Prentice-Hall, 2002.

18. Miaou SG: Digital Image Processing Using Matlab. Chuan Hwa Books Co. Ltd., Taiwan, 1999. 